\title{
Observable Deviations from Homogeneity in an Inhomogeneous Universe
}

\author{
John T. Giblin, Jr ${ }^{1,2}$, James B. Mertens ${ }^{2}$, and Glenn D. Starkman ${ }^{2}$ \\ ${ }^{1}$ Department of Physics, Kenyon College, $201 \mathrm{~N}$ College Rd, Gambier, OH 43022 and \\ ${ }^{2}$ CERCA/ISO, Department of Physics, Case Western Reserve University, 10900 Euclid Avenue, Cleveland, OH 44106
}

\begin{abstract}
How does inhomogeneity affect our interpretation of cosmological observations? It has long been wondered to what extent the observable properties of an inhomogeneous universe differ from those of a corresponding Friedman-Lemaitre-Robertson-Walker (FLRW) model, and how the inhomogeneities affect that correspondence. Here, we use numerical relativity to study the behavior of light beams traversing an inhomogeneous universe and construct the resulting Hubble diagrams. The universe that emerges exhibits an average FLRW behavior, but inhomogeneous structures contribute to deviations in observables across the observer's sky. We also investigate the relationship between angular diameter distance and the angular extent of a source, finding deviations that grow with source redshift. These departures from FLRW are important path-dependent effects with implications for using real observables in an inhomogeneous universe such as our own.
\end{abstract}

\section{INTRODUCTION}

The field of cosmology has always relied on the cosmological principle: the Universe is homogeneous and isotropic. This principle has been used to construct the dynamical models with which we understand and interpret the evolution of the Universe; and in the context of this principle we define and measure cosmological parameters. However, as cosmological measurements reach percent-level precision, the effects of inhomogeneities in the Universe become increasingly important when comparing observations to models. The field of cosmology has typically studied these effects using a perturbative approach, with inhomogeneities described and modeled by a linearized-gravity approximation [1, 2]. Additionally, semi-analytic models have been used to study effects of inhomogeneities on observables in specific spacetimes, such as Swiss Cheese or Lindquist-Wheeler metrics $[3] 6$.

Just a short time ago, the dynamics of cosmological dust spacetimes were examined in full 3+1-dimensional general relativity (GR) for the first time by solving the Einstein field equations numerically $7 \sqrt{9}$. These studies and others such as [10 12] have, so far, focused on mathematical and numerical properties of the spacetimes, rather than the impact of inhomogeneities on cosmological observables. An open question therefore remained: can we compute predictions for observables and demonstrate the necessity and power of these numerical simulations?

In this work, we present the first calculation of observable quantities in cosmology using the full, unconstrained framework of numerical relativity for a pure "dust" spacetime containing inhomogeneities on large scales a framework consistent with the work presented in [7 9]. We follow light beams along null geodesics through a toy universe and integrate the optical scalar equations along these geodesics, garnering information about angular diameter distances and photon redshifts as the universe evolves. Using these observables, we construct the resulting Hubble diagrams, plotting the distance modulus versus redshift, and compare the results to the Friedman-
Lemaitre-Robertson-Walker (FLRW) model.

We thus extend the previous numerical studies in GR by examining the properties of photons traversing these spacetimes. A schematic of this scenario is depicted in Fig. 1. In general, we find good agreement between the averaged observables and corresponding FLRW model; however, there are departures along individual paths, and when observers or sources are located in under-densitites or over-densities.

The demonstration of this method by way of drawing Hubble diagrams is the main result of this work. We explore the ability of our code to resolve effects that man-

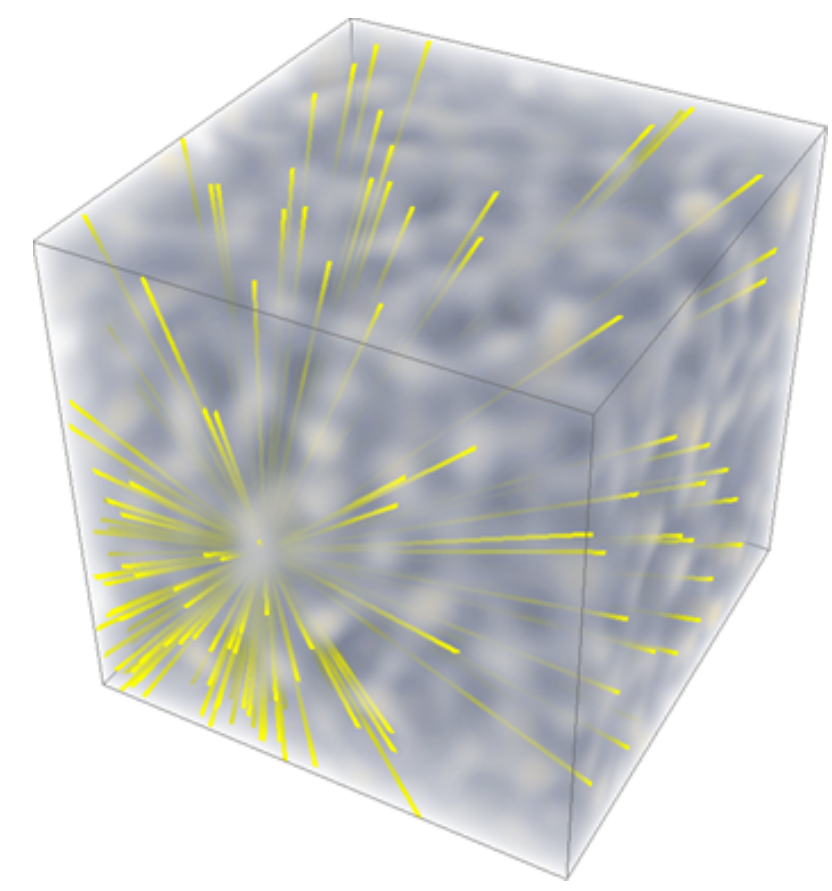

FIG. 1: A depiction of raytracing through a spacetime with density fluctuations. Shades of lighter gray represent denser regions of space, and darker colors represent less dense regions. In a time-reversed sense, light rays (yellow) originate from an observer located in the volume and propagate outward along null geodesics. 
ifest themselves in Hubble diagrams due to observers being located in over-dense or under-dense regions [13 15]. We additionally demonstrate the ability of this method to examine the relationship between different distance measures, such as luminosity distance and observed angular extent, probing discrepancies that might arise due to inhomogeneities. While our model attempts to mimic our Universe on large scales, we do not yet resolve smallscale structure, and that the amplitude of fluctuations is not observationally motivated. Nevertheless, we hope to be able to model these more realistically in the future.

We begin in Section II A by briefly reviewing the formulation of numerical relativity used in this work, and describing, in Sections IIC and II B the formalism we use to integrate photon geodesics. We then provide details of the numerical technique used to integrate these equations in Section III. Finally, in Section IV] we present results from a series of cosmologically-motivated simulations, and remark on Etherington's reciprocity relation and the ability of our technique to probe outstanding questions in cosmology.

\section{EVOLUTION OF OBSERVABLES IN A GENERAL SPACETIME}

Numerical relativity has seen a number of breakthroughs in the past two decades, notably the development of formulations capable of stable long-term evolution. We take advantage of these advancements, and once again [8] apply them in a cosmological setting. We also write the geodesic equations and optical-scalar equations in a form suitable for integration in our numerical spacetime.

\section{A. The BSSN formulation}

The BSSN formulation [16, 17] is a variant of a $3+1$ decomposition with important modifications for improving the numerical stability of the system. For a review or for further details, see eg. [18, 19]. In this formulation, the metric is decomposed as

$$
g_{\mu \nu}=\left(\begin{array}{cc}
-\alpha^{2}+\beta_{l} \beta^{l} & \beta_{i} \\
\beta_{j} & e^{4 \phi} \bar{\gamma}_{i j}
\end{array}\right),
$$

where $\gamma_{i j}=e^{4 \phi} \bar{\gamma}_{i j}$ is the spatial metric with $\operatorname{det}\left(\bar{\gamma}_{i j}\right)=1$, and $\alpha$ and $\beta^{i}$ are the lapse and shift respectively. The metric obeys the BSSN evolution equations,

$$
\begin{aligned}
\partial_{t} \phi= & -\frac{1}{6} \alpha K+\beta^{i} \partial_{i} \phi+\frac{1}{6} \partial_{i} \beta^{i} \\
\partial_{t} \bar{\gamma}_{i j}= & -2 \alpha \tilde{A}_{i j}+\beta^{k} \partial_{k} \bar{\gamma}_{i j}+\bar{\gamma}_{i k} \partial_{j} \beta^{k} \\
& +\bar{\gamma}_{k j} \partial_{i} \beta^{k}-\frac{2}{3} \bar{\gamma}_{i j} \partial_{k} \beta^{k} \\
\partial_{t} K= & -\gamma^{i j} D_{j} D_{i} \alpha+\alpha\left(\tilde{A}_{i j} \tilde{A}^{i j}+\frac{1}{3} K^{2}\right) \\
& +4 \pi \alpha(\rho+S)+\beta^{i} \partial_{i} K
\end{aligned}
$$

$$
\begin{aligned}
\partial_{t} \tilde{A}_{i j}= & e^{-4 \phi}\left(-\left(D_{i} D_{j} \alpha\right)+\alpha\left(R_{i j}-8 \pi S_{i j}\right)\right)^{T F} \\
& +\alpha\left(K \tilde{A}_{i j}-2 \tilde{A}_{i l} \tilde{A}_{j}^{l}\right)+\beta^{k} \partial_{k} \tilde{A}_{i j} \\
& +\tilde{A}_{i k} \partial_{j} \beta^{k}+\tilde{A}_{k j} \partial_{i} \beta^{k}-\frac{2}{3} \tilde{A}_{i j} \partial_{k} \beta^{k}
\end{aligned}
$$

with source terms $\rho, S, S_{j}$, and $S_{i j}$ projections of the stress-energy tensor onto the spatial slice. A key to the numerical stability of the system is the introduction of a redundant set of auxiliary variables, contractions of the Christoffel symbol of the conformal metric $\bar{\gamma}_{i j}, \bar{\Gamma}^{i} \equiv \bar{\gamma}^{j k} \bar{\Gamma}_{j k}^{i}$, which are used to compute the Ricci tensor. These are also dynamically evolved,

$$
\partial_{t} \bar{\Gamma}^{i}=2\left(\bar{\Gamma}_{j k}^{i} \tilde{A}^{j k}-\frac{2}{3} \bar{\gamma}^{i j} \partial_{j} K-8 \pi \bar{\gamma}^{i j} S_{j}+6 \tilde{A}^{i j} \partial_{j} \phi\right)
$$

In this scheme, the extrinsic curvature, $K_{i j}$, has been decomposed into a conformally related trace-free part, $\tilde{A}_{i j}$, and trace, $K$, as $K_{i j}=e^{4 \phi} \tilde{A}_{i j}+\frac{1}{3} \gamma_{i j} K$. Although our code allows for an arbitrary gauge choice, synchronous gauge $\left(\alpha=1\right.$ and $\left.\beta^{i}=0\right)$ is applied in this work.

A pressureless, $w=0$, perfect fluid is used to model the matter component of this universe on large scales, consistent with a cold-dark matter model. The general equations of motion for such a fluid require integration techniques tailored to the problem, such as finite-volume methods 20], phase-space methods 21, or N-body techniques 22]. However, in synchronous gauge, the equations of motion simplify to

$$
\partial_{t}\left(\gamma^{1 / 2} \rho\right)=0
$$

for a fluid with no initial coordinate velocity and density $\rho$. Although multistreaming - or the crossing of fluid elements - cannot be resolved using this formulation due to the formation of coordinate singularities, this does not present an issue on the large scales examined in this work. We can therefore choose an initial conformal density $\gamma^{1 / 2} \rho$ that will not evolve over the course of the simulation. For this choice of coordinates, the remaining matter source terms in the BSSN Equations are $S=S_{j}=S_{i j}=0$.

\section{B. Propagation along null geodesics}

While the BSSN formalism allows us to evolve the metric, we wish also to compute measurable quantities. We therefore derive the equations of motion for particles traveling along geodesics. Although we are particularly interested in null geodesics, the equations presented in this section can also be applied to massive particles.

Adopting the notation of (and loosely following) 23. we begin by noting that the equations describing propagation along a geodesic are often parametrized by an affine variable, $\lambda$. We will need to re-parametrize these equations in terms of the coordinate time $t$ and variables 
from a $3+1$ decomposition. The 4 -momentum, $p^{\mu}$, for a particle following a geodesic is

$$
\frac{\mathrm{d} X^{\mu}}{\mathrm{d} \lambda}=p^{\mu}
$$

for an affine parameter $\lambda$ that parametrizes the particle's path. In order to work in terms of $3+1$ quantities, the 4-momentum is decomposed and written in terms of quantities according to observers on a spatial slice. This is accomplished by writing the 4-momentum in terms of a piece parallel to the unit normal to the slices, $p_{\|}^{\mu} \| n^{\mu}$, where the normal is $n^{\mu}=\left(\alpha^{-1},-\alpha^{-1} \beta^{i}\right)$, as well as a transverse piece proportional to the velocity according to a normal observer, $p_{\perp}^{\mu} \propto V^{\mu}$.

We then define the energy of the particle according to a normal observer as $E \equiv-n_{\mu} p^{\mu}$. The zero-component of the 4-momentum can be related to $E$ by noticing that

$$
n_{\mu} \mathrm{d} X^{\mu}=n_{\mu} p^{\mu} d \lambda
$$

which is just $E / \alpha=p^{0}$. We can now decompose the 4-momentum as

$$
\begin{aligned}
p_{\|}^{\mu} & =p^{\mu}-p_{\perp}^{\mu}=E n^{\mu} \\
p_{\perp}^{\mu} & =p^{\mu}-E n^{\mu} \equiv E V^{\mu},
\end{aligned}
$$

where $p^{\mu}=E\left(n^{\mu}+V^{\mu}\right)$. Because the velocity vector $V^{\mu}$ has no component in the direction normal to the spatial slices $\left(n_{\mu} V^{\mu}=0\right)$, we can choose to write it as $V^{\mu}=$ $\left(0, V^{i}\right)$, with no time component.

We now wish to obtain evolution equations for $E$ and $V^{i}$ in terms of a coordinate time $t$ rather than affine parameter. We can do so using the geodesic equation,

$$
\frac{\mathrm{d}^{2} X^{\mu}}{\mathrm{d} \lambda^{2}}=-\Gamma_{\alpha \beta}^{\mu} \frac{\mathrm{d} X^{\alpha}}{\mathrm{d} \lambda} \frac{\mathrm{d} X^{\beta}}{\mathrm{d} \lambda}
$$

for the position of a particle, $X^{\mu}$, along a path parametrized by $\lambda$. This can be written in a form wellsuited for integrating the $3+1$ variables, resulting in a closed set of equations of motion:

$$
\begin{aligned}
\frac{1}{E} \frac{\mathrm{d}}{\mathrm{d} t} E= & \alpha K_{i j} V^{j} V^{k}-V^{j} \partial_{j} \alpha \\
\frac{\mathrm{d} X^{i}}{\mathrm{~d} t}= & \alpha V^{i}-\beta^{i} \\
\frac{\mathrm{d} V^{i}}{\mathrm{~d} t}= & \alpha V^{j}\left(V^{i} \partial_{j} \ln \alpha-K_{j k} V^{k} V^{i}+2 K_{j}^{i}\right. \\
& \left.-{ }^{(3)} \Gamma_{j k}^{i} V^{k}\right)-\gamma^{i j} \partial_{j} \alpha-V^{j} \partial_{j} \beta^{i}
\end{aligned}
$$

\section{Angular diameter distances and the optical scalar equations}

The angular diameter distance, $D_{A}$, is a standard distance measure used in cosmology, with the desirable property that it can be directly measured or computed for distant sources. It is also related to the luminosity distance: $D_{A}=(1+z)^{2} D_{L}$. This statement, known as the Etherington Reciprocity Theorem or distance duality relationship [24, 25], is a geometric result, and holds so long as photon number is conserved and all photons in the beam "feel" the same metric - meaning the beam width is small compared to distances over which the metric varies. This small beam limit should be a good approximation for point-like sources such as supernovae, but not necessarily for beams with a large angular extent, such late-universe baryon acoustic oscillations, or degree-scale CMB fluctuations. A more thorough discussion can be found in other works, eg. [26].

In order to compute angular diameter distances, we must track the cross-sectional area of a light beam as it propagates through space. We accomplish this using the Sachs optical-scalar equations, rewritten in a form well-suited to both numerical integration and extraction of cosmological parameters. These equations track the width of a beam $\ell$, its time-derivative $\varphi$, the shear rate of the beam $\sigma$, and basis vectors, known as screen vectors, spanning the plane of the beam area, $s_{1}^{\mu}$ and $s_{2}^{\mu}$. The evolution equations (briefly elaborated upon in Appendix $\mathrm{A}$ and Appendix B are

$$
\begin{aligned}
p^{0} \frac{\mathrm{d}}{\mathrm{d} t} \varphi & =\ell\left(\mathcal{R}-\sigma_{R}^{2}-\sigma_{I}^{2}\right) \\
p^{0} \frac{\mathrm{d}}{\mathrm{d} t} \ell & =\varphi \\
p^{0} \frac{\mathrm{d}}{\mathrm{d} t} \bar{\sigma}_{R} & =\ell^{2} \Re[\mathcal{W}] \\
p^{0} \frac{\mathrm{d}}{\mathrm{d} t} \bar{\sigma}_{I} & =\ell^{2} \Im[\mathcal{W}],
\end{aligned}
$$

with $\bar{\sigma}=\ell^{2} \sigma$. The Ricci optical scalar $\mathcal{R}$ is given by

$$
\mathcal{R}=-4 \pi T_{\mu \nu} p^{\mu} p^{\nu}
$$

and the real and imaginary pieces of the Weyl scalar $\mathcal{W}$ are

$$
\begin{aligned}
& \Re[\mathcal{W}]=-\frac{1}{2} C_{\mu \nu \rho \sigma}\left(s_{1}^{\mu} s_{1}^{\sigma}-s_{2}^{\mu} s_{2}^{\sigma}\right) p^{\nu} p^{\rho} \\
& \Im[\mathcal{W}]=C_{\mu \nu \rho \sigma} s_{1}^{\mu} p^{\nu} p^{\rho} s_{2}^{\sigma} .
\end{aligned}
$$

The screen vectors evolve according to

$$
\begin{aligned}
\frac{\mathrm{d}}{\mathrm{dt}} s_{A}^{\mu}= & s_{A}^{j} V^{i}\left(\gamma_{j k} \frac{\mathrm{d}}{\mathrm{dt}} V^{k}-3 V^{k} K_{j k}+V_{l} V^{k} \Gamma_{k j}^{l}\right) \\
& -\Gamma_{k l}^{i} V^{k} s_{A}^{l}+\gamma^{i j} K_{j k} s_{A}^{k} .
\end{aligned}
$$

These equations are subject to boundary conditions at the point of observation, namely that the beam area has converged, $\ell\left(t_{o b s}\right)=0$, the observer views the beam as subtending some solid angle $\varphi\left(t_{o b s}\right)=\sqrt{\Omega_{o b s}}$, and the shear rate is $\sigma=0$ [3]. In terms of these variables, the angular diameter distance to a source that emitted light at some time $t_{e m}$, seen by an observer as subtending a solid angle $\Omega_{o b s}$ at time $t_{o} b s$, is $D_{A}=\ell\left(t_{e m}\right) / \varphi\left(t_{o b s}\right)$. 
We conclude this section by writing the relationship between various quantities defined so far, and corresponding FLRW quantities for a completely homogeneous spacetime, in Table I.

\begin{tabular}{c|c|c} 
Quantity & FLRW Notation & Our Notation \\
\hline Scale factor & $a$ & $e^{2 \phi}$ \\
Hubble parameter & $H$ & $-K / 3$ \\
Photon redshift & $z$ & $E-1$ \\
Angular diameter distance & $D_{A}$ & $\ell_{\text {em }} / \varphi_{\text {obs }}$
\end{tabular}

TABLE I: Translation between variables in a completely homogeneous dust universe.

\section{NUMERICAL METHOD DETAILS}

As we are interested in examining the dynamics of photon geodesics converging at an observer, we will need to ensure our boundary conditions at the observer are satisfied, or that beams have converged at a particular spacetime point. The most straightforward and consistent way to implement this is to integrate a system forward from some initial state to a future observation time, and then trace photon paths while integrating the system of equations backwards in time. Our code implements precisely this, allowing us to evolve the system from some cosmological initial conditions to a future state, change the sign of the timestep, and continue integrating both the metric and photon geodesics. The cosmological initial conditions we use consist of a Gaussian-random realization of a matter field with a cosmologically-motivated matter power spectrum in a periodic spacetime,

$$
P_{k}^{\rho}=\frac{4 P_{*}}{3} \frac{k / k_{*}}{1+\frac{1}{3}\left(k / k_{*}\right)^{4}} .
$$

We cut $P_{k}^{\rho}$ off at some finite $k_{\text {cutoff }}$ in order to reduce fluctuations on scales where grid effects become important, so that fluctuations are resolved by sufficiently many points. Further details of this spacetime can be found in [8]. In order to specify initial conditions for light rays, we pick random outgoing directions from a specified point, and integrate along null geodesics in these directions backwards in time.

Integrating backwards not only allows us to ensure light rays converge at an observer, but also allows us to verify that we evolve back to the initial state imposed at the beginning of the simulation. We find that our code passes this check to within a small, expected level of error. While of importance in our work, such a technique would not necessarily be suitable for simulations without periodic boundary conditions, as information about the state of the metric may propagate outside the volume of interest when using, for example, damping boundary conditions. This method may also fail for hydrodynamical calculations in the presence of shocks.
Because we integrate through a discretized spacetime, we also need to compute metric quantities between grid points, requiring an interpolation scheme. In this code we utilize simple linear interpolation, accurate only to $\mathcal{O}\left(\Delta x^{2}\right)$. In principle this could be improved, as the metric and matter fields are evolved using an $\mathcal{O}\left(\Delta x^{8}\right)$ scheme, however this level of accuracy is not found to be necessary here. Accumulated error for quantities integrated along geodesics is therefore $O(\Delta x)$, the dominant contribution to the error in our results. For cosmological runs (such as presented in [7]), we see convergence as expected given the interpolation and integration order used to follow photon trajectories. Further details of the code we use to evolve the spacetime can be found in [8].

We use results from simulations run at three different resolutions: $N^{3}=128^{3}, N^{3}=144^{3}$ and $N^{3}=160^{3}$. We then use a standard numerical technique, Richardson extrapolation [27, 28, in order to test convergence, to obtain results at higher order, and to obtain error estimates.

As a final note on error, the statistical uncertainty in computed means scales as $1 / \sqrt{N_{\gamma}}$, with $N_{\gamma}$ the number of traced rays. In general, we will be tracing $N_{\gamma}=10^{3}$ rays for each observer, so we expect statistical error in the means to be a few per-cent of the variance. Numerical error in computed quantities is found to be of order a part in $10^{6}$, significantly smaller than this statistical error.

\section{RESULTS: COMPUTING OBSERVABLES}

In this section we present results from following light beams through a series of simulated cosmological spacetimes similar to the fiducial model presented in [7]. We begin by examining the properties of observables in our model, and describe the effects of the inhomogeneities compared to a corresponding FLRW model, or a homogeneous universe containing only pressureless matter (eg., $\left.\Omega_{m}=1\right)$. The initial density fluctuations in these runs are around $\sigma_{\rho} / \rho=0.1$, which grow to $\sigma_{\rho} / \rho=0.2$ on the spatial slice containing our observers. On average, we see that our simulations reproduce the FLRW universe extremely well; however there are significant deviations along individual paths and when observers or sources are preferentially located in under- or over-dense regions.

We first remark on the case of an observer whose local density corresponds closely to the average value on a spatial slice. In this case, we find light beams traversing individual geodesics trace out a uniform distribution of angular diameter distances, the mean of which closely tracks the FLRW expectation at all redshifts we examine. In Fig. 2 we present a Hubble diagram as would be computed by such an average observer, after converting the angular-diameter distances to apparent magnitudes, $m-M=5 \log _{10}\left(D_{A}(1+z)^{-2} / 10 \mathrm{pc}\right)$.

In Fig. 3, we present the corresponding residual Hubble diagram. In this diagram we subtract the distance modulus for a matter-dominated FLRW model from the 


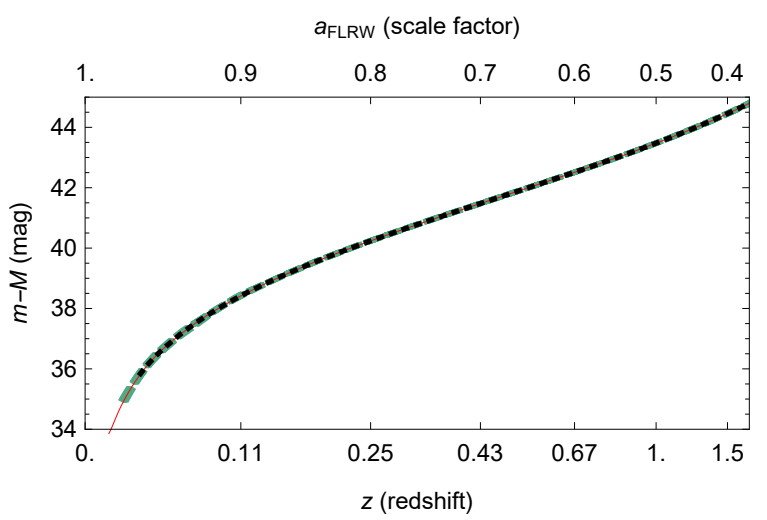

FIG. 2: A Hubble diagram according to an 'average' observer. The black dotted line indicates the prediction from a pure-FLRW model, many green dashed lines are derived from angular diameter distances computed along individual lines of sight, and the red line is the average along all individual lines of sight. The different lines are nearly indistinguishable.

distance modulus we calculate for our model for each value of $z$. Of note, there is some ambiguity in choosing which FLRW model to compare to. For example, the FLRW model that agrees with the conformal average density at a particular time will not necessarily agree at a later time, and will also not necessarily agree with the FLRW model that agrees with the (conformal) average expansion rate. For an increasingly inhomogeneous universe these differences may become important, however for our simulations, the differences are largely unimportant. We thus compare to a pure-FLRW model for which the expansion rate agrees with the conformally averaged expansion rate of the initial simulation hypersurface.

In the residual diagram, we find that despite fluctuations along individual paths, there is close agreement between the average angular diameter distance and corresponding FLRW model at most of the redshifts we probe. Of significance, at very low redshift $(z \lesssim 0.1)$, there are sizable deviations. These arise primarily due to the local structure of the universe: although this observer is situated at a location of near-average density, they are nevertheless near both over-dense and under-dense patches, which in turn affect angular-diameter measurements. Averaged across many such observers, these local effects can disappear; however for an individual observer we do not find this to be the case. At larger redshifts, we find agreement between averaged quantities and the corresponding FLRW model to within the uncertainty of our model.

We could compare this behavior to semi-analytic models, for example Swiss-Cheese universes, using the DyerRoeder approximation. To an extent, our findings contrast the ideas behind such models, which posit that light beams will undergo less focusing as they intercept less matter (for a given average density) in "lumpier" universes. However, our current simulations are in a regime that should be well-described by linear theory, or

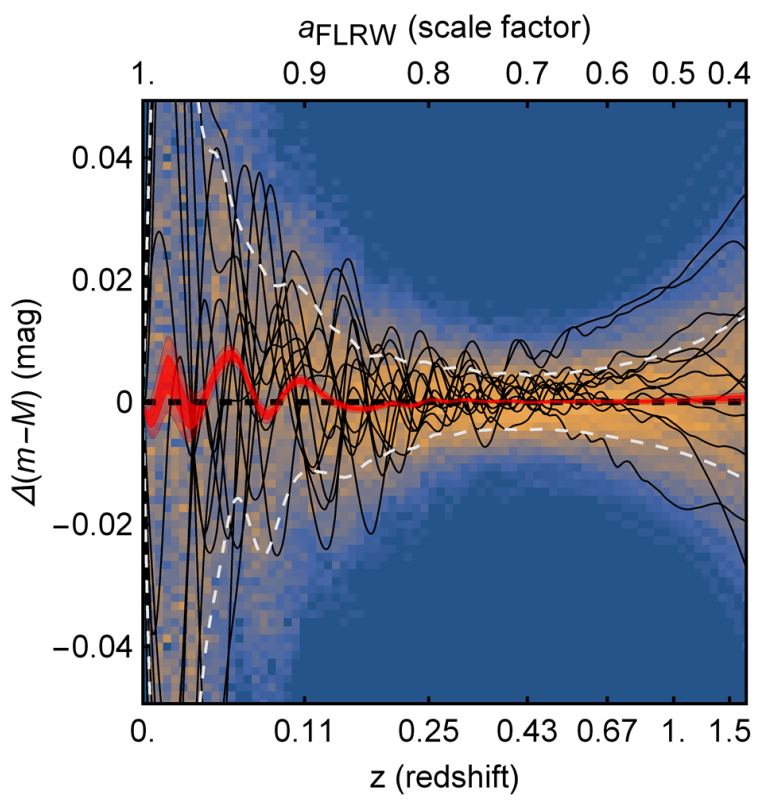

FIG. 3: A residual Hubble diagram according to an 'average' observer. Thin black lines depict angular diameter distances along several particular lines of sight for this observer. The black dashed line indicates the prediction from a pure-FLRW model. The solid red line indicates the average angular diameter distance of these rays, for which we see agreement with FLRW. The statistical error - standard error in the mean - is shown at the one and two 'sigma' level, indicated by semi-transparent red shading, so the width of the red curve is indicative of error. White dashed lines indicate the standard deviation of the distribution of magnitudes. Finally, the background shows a histogram of the magnitudes along all integrated lines of sight as a function of redshift.

a regime in which fluctuations are normally distributed around the FLRW solution, and thus average away. These models also suggest the shear terms are negligible, something we find approximate agreement with; the size of the shear terms relative to the Ricci optical scalar is $\mathcal{R} /\left|\sigma^{2}\right| \sim \mathcal{O}(0.01 \%)$. Nevertheless, once collapsed structure is better resolved by future work, investigating these comparisons will be an interesting and important task.

We next examine effects due to an observer being situated in a local over-dense or under-dense region. In synchronous gauge, especially at early times, fluctuations in the metric are directly sourced by fluctuations in the density of the universe. In our simulations, and in our coordinates, the fluid is at rest throughout the simulation, so photons passing through over-dense or under-dense regions will be redshifted according to how the metric has responded to the over- or under-density. In synchronous gauge, this can be interpreted as an analogue to a latetime integrated Sachs-Wolfe effect, or Rees-Sciama effect. In a gauge where the fluid is not co-moving, at least some of this effect may interpreted as due to peculiar velocities. In Fig. 4 we see such an effect for an observer in a local over-density. We note that the effect of an observer sitting in a local under-density is similar but opposite in 


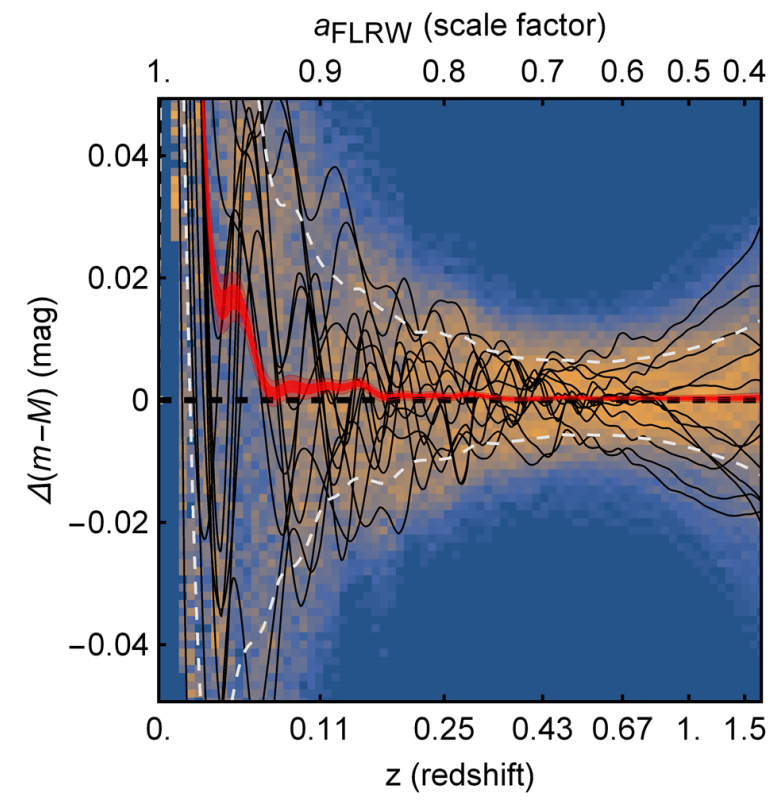

FIG. 4: A Hubble diagram as in figure 3 created by raytracing through the simulated universe, but for an observer located in an overdensity. Effects due to local structure are manifest at low redshift.

magnitude and can mimic the behavior of a cosmological constant. The idea that we are situated in a large void has been explored in great detail in the past, see eg. 14, 15. While such an effect is unlikely to explain cosmic acceleration, it could nevertheless result in local measurements of cosmological parameters that do not reflect more global properties of the spacetime.

In a similar vein, we could use our technique to explore effects due to the tendency of observable sources to lie in regions of higher density. One might expect to see the average deviation from FLRW affected by a Sachs-Wolfetype effect, as photons not only climb out of gravitational potential wells, but undergo additional Ricci focusing due to the presence of a local overdensity at the source, resulting in a slightly diminished inferred angular diameter distance. Preliminary explorations of this effect suggest this could be an interesting future topic of study.

We conclude by examining the ability of our technique to explore differences in inferred angular diameter distances for objects of large angular extent due to inhomogenities. Such effects can manifest themselves in observations of extended structures, for example degreescale CMB fluctuations or Baryon-Acoustic-Oscillationscale structures. In principle, a similar calculation can be used to directly probe breakdowns of the Etherington Reciprocity theorem due to finite beam effects. Violations of this theorem are of particular interest due to their ability to probe physics affecting the underlying assumptions of the theorem, and have thus become increasingly well constrained [29.

As a simplistic experiment, consider two pointlike sources with a known luminosity, part of a structure of known size, so the distance between them is known. Thus the luminosity distance is known to each pointlike source independently, and the angular diameter distance to the aggregate object can be inferred. In the limit that the point sources are infinitesimally separated, the relationship between the distance measures should obey Etherington's reciprocity theorem. However as the source separation - and therefore the separation between rays increases, the inferred angular diameter distance may no longer agree with the luminosity distances due to different lines of sight probing different metric potentials. Thus, the inferred angular diameter distance will disagree with at least one of the luminosity distances.

In Fig. 5, we present the difference in angular diameter distances computed by integrating the optical scalar equations, to an aggregate 'source' with finite angular extent, assuming the source is located at a fixed redshift. We find very small deviations of distance measures on average and for beams with small area, however individual measurements demonstrate appreciable deviations, particularly on large angular scales. For actual observations, the situation may differ in several regards. For example, if sources used as standard rulers lie in similar gravitational potentials, the particular contribution of gravitational redshift or peculiar velocity may be reduced.

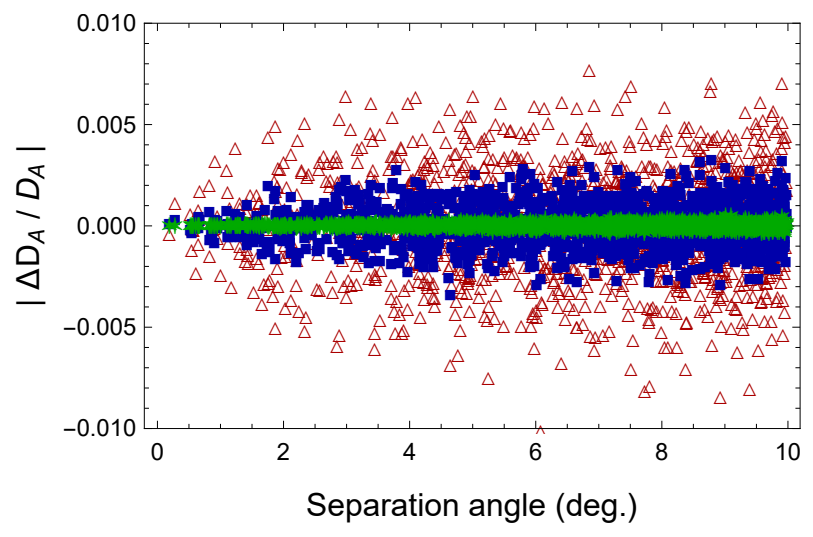

FIG. 5: Distribution of fractional differences in angular diameter distance, $\Delta D_{A} / D_{A}$, for infinitesimal beams separated by an angle. Beams are integrated out to sources at $z=0.1$ (green stars), $z=0.5$ (blue squares), and $z=1.0$ (red triangles). At small separation, angular diameter distances agree; however at larger separation, increasing disagreement is found.

\section{CONCLUSIONS AND DISCUSSION}

The results presented here are the next step in an important advancement of numerical techniques that allows us to directly integrate the full Einstein field equations and the optical scalar equations in an arbitrary spacetime. This in turn enables computation of cosmological observables and directly measurable quantities. As numerical relativity continues to find new applications 
in cosmology, long-standing questions will be resolved in regimes where non-linearities are significant. Determining the relevance of, and precisely quantifying these effects in relativistic models will be an important future task for this field.

Here we have examined the ability of techniques from numerical relativity to probe aspects of cosmology where precise estimates are required. We consider a universe with large-scale inhomogeneities, so while we expect perturbation theory and approximation schemes such as Dyer-Roeder may accurately describe the effects we observe, it is encouraging to see the well-understood physical effects manifesting themselves in this model.

Numerous applications of numerical relativity can be made in future studies, and it will be interesting to see the direction these studies take. For example, while statistical studies can offer general insight into physical processes, it may also be possible to model specific systems to better understand dynamics and extract cosmological information.

\section{ACKNOWLEDGMENTS}

We would like to thank Thomas Baumgarte for a series of very valuable conversations, as well as Stuart Shaprio and Lorenzo Sorbo for conversations that helped shape this work. We are also grateful for feedback from Pierre Fleury on an earlier draft of this manuscript. JTG is supported by the National Science Foundation, PHY1414479; JBM and GDS are supported by a Department of Energy grant DE-SC0009946 to CWRU. The simulations in this work made use of the High Performance Computing Resource in the Core Facility for Advanced Research Computing at Case Western Reserve University, and of hardware provided by the National Science Foundation, the Research Corporation for Science Advancement, and the Kenyon College Department of Physics.
[1] F. Bernardeau, S. Colombi, E. Gaztanaga, and R. Scoccimarro, Phys. Rept. 367, 1 (2002), astro-ph/0112551.

[2] J. Adamek, D. Daverio, R. Durrer, and M. Kunz, Nature Phys. 12, 346 (2016), 1509.01699.

[3] P. Fleury, H. Dupuy, and J.-P. Uzan, Phys. Rev. D87, 123526 (2013), 1302.5308.

[4] P. Fleury, JCAP 1406, 054 (2014), 1402.3123.

[5] K. Bolejko and P. G. Ferreira, JCAP 1205, 003 (2012), 1204.0909.

[6] T. Clifton, P. G. Ferreira, and K. O'Donnell, Phys. Rev. D85, 023502 (2012), 1110.3191.

[7] J. T. Giblin, J. B. Mertens, and G. D. Starkman, Phys. Rev. Lett. 116, 251301 (2016), 1511.01105.

[8] J. B. Mertens, J. T. Giblin, and G. D. Starkman, Phys. Rev. D93, 124059 (2016), 1511.01106.

[9] E. Bentivegna and M. Bruni, Phys. Rev. Lett. 116, 251302 (2016), 1511.05124.

[10] C.-M. Yoo, H. Okawa, and K.-i. Nakao, Phys. Rev. Lett. 111, 161102 (2013), 1306.1389.

[11] E. Bentivegna and M. Korzynski, Class. Quant. Grav. 30, 235008 (2013), 1306.4055.

[12] C.-M. Yoo and H. Okawa, Phys. Rev. D89, 123502 (2014), 1404.1435.

[13] M. J. Rees and D. W. Sciama, Nature 217, 511 (1968).

[14] J. Garcia-Bellido and T. Haugboelle, JCAP 0804, 003 (2008), 0802.1523.

[15] T. Biswas, A. Notari, and W. Valkenburg, JCAP 1011, 030 (2010), 1007.3065.

[16] T. W. Baumgarte and S. L. Shapiro, Phys. Rev. D59, 024007 (1999), gr-qc/9810065.

[17] M. Shibata and T. Nakamura, Phys. Rev. D52, 5428 (1995).

[18] V. Cardoso, L. Gualtieri, C. Herdeiro, and U. Sperhake, Living Rev. Relativity 18, 1 (2015), 1409.0014.

[19] T. W. Baumgarte and S. L. Shapiro, Numerical Relativity: Solving Einstein's Equations on the Computer (Cambridge University Press, Cambridge, UK, 2010).

[20] M. COLOMBEAU, SIAM Journal on Numerical Analy- sis 48, 1900 (2010), ISSN 00361429, URL http://www. jstor.org/stable/41149030.

[21] F. A. Rasio, S. L. Shapiro, and S. A. Teukolsky, Astrophys. J. 344, 146 (1989).

[22] I. Olabarrieta, Ph.D. thesis, British Columbia U. (2000), gr-qc/0012059, URL http://alice.cern.ch/format/ showfull? sysnb=2234989.

[23] F. H. Vincent, E. Gourgoulhon, and J. Novak, Class. Quant. Grav. 29, 245005 (2012), 1208.3927.

[24] I. M. H. Etherington, General Relativity and Gravitation 39, 1055 (2007), ISSN 1572-9532, URL http://dx.doi. org/10.1007/s10714-007-0447-x

[25] G. F. R. Ellis, General Relativity and Gravitation 39, 1047 (2007), ISSN 1572-9532, URL http://dx.doi.org/ 10.1007/s10714-006-0355-5.

[26] S. Seitz, P. Schneider, and J. Ehlers, Class. Quant. Grav. 11, 2345 (1994), astro-ph/9403056.

[27] W. H. Press, S. A. Teukolsky, W. T. Vetterling, and B. P. Flannery, Numerical Recipes 3rd Edition: The Art of Scientific Computing (Cambridge University Press, New York, NY, USA, 2007), 3rd ed., ISBN 0521880688, 9780521880688.

[28] M. Alcubierre, Introduction to $3+1$ numerical relativity, International series of monographs on physics (Oxford Univ. Press, Oxford, 2008), URL https : //cds . cern.ch/ record/1138167.

[29] S. Rasanen, J. Valiviita, and V. Kosonen, JCAP 1604, 050 (2016), 1512.05346.

[30] R. K. Sachs, Proc. Roy. Soc. Lond. A264, 309 (1961).

[31] E. Newman and R. Penrose, J. Math. Phys. 3, 566 (1962).

[32] P. Fleury, Ph.D. thesis, Paris, Inst. Astrophys. (2015), 1511.03702, URL https://inspirehep.net/ record/1404129/files/arXiv:1511.03702.pdf 


\section{A. ANGULAR DIAMETER DISTANCES}

The optical focusing equations [30],

$$
\begin{aligned}
\frac{\mathrm{d}}{\mathrm{d} \lambda} \theta+\theta^{2}+|\sigma|^{2} & =\mathcal{R} \\
\frac{\mathrm{d}}{\mathrm{d} \lambda} \sigma+2 \theta \sigma & =\mathcal{W},
\end{aligned}
$$

describe the evolution of a beam with infinitesimal area. The standard variable $\theta=1 / 2 A \mathrm{~d} A / \mathrm{d} \lambda$ describes the rate of expansion of a beam's area $A$ (and is not an angle). The equations of motion we use, however, work in terms of the rooted beam area $\ell=\sqrt{A}$, for which $\theta=1 / \ell \mathrm{d} \ell / \mathrm{d} \lambda$. The quantity $\sigma$ is a complex scalar, loosely describing the shear rate of a light beam up to additional geometric factors. The Weyl and Ricci optical scalars, $\mathcal{W}$ and $\mathcal{R}$ respectively, are

$$
\begin{aligned}
\mathcal{W} & =-\frac{1}{2} C_{\mu \nu \rho \sigma}\left(s_{1}^{\mu}-i s_{2}^{\mu}\right) p^{\nu} p^{\rho}\left(s_{1}^{\sigma}-i s_{2}^{\sigma}\right) \\
\mathcal{R} & =-4 \pi T_{\mu \nu} p^{\mu} p^{\nu}=-\frac{1}{2} R_{\mu \nu} p^{\mu} p^{\nu}
\end{aligned}
$$

for screen vectors $s_{1}^{\mu}, s_{2}^{\mu}$, and Weyl tensor $C_{\mu \nu \rho \sigma}$. The Weyl scalar here is analogous to those from the NewmanPenrose formalism for identifying gravitational radiation 31; however the vectors used to compute the Weyl scalar are comprised of a single null vector $p^{\mu}$ and the screen vectors rather than a null tetrad.

The screen vectors obey the following orthogonality relationships: $g_{\mu \nu} s_{N}^{\nu} p^{\mu}=0$ (normal to the photon 4vector), $g_{\mu \nu} s_{N}^{\nu}\left(q^{\mu}-U^{\mu}\right) \equiv g_{\mu \nu} s_{N}^{\nu} d^{\mu}=0$ (normal to the 'direction of observation' $d^{\mu}$ and thus observer 4-velocity $U^{\mu}$ ), and $g_{\mu \nu} s_{1}^{\mu} s_{2}^{\nu}=0$, and $g_{\mu \nu} s_{1}^{\mu} s_{1}^{\nu}=g_{\mu \nu} s_{2}^{\mu} s_{2}^{\nu}=1$ (orthonormality of $s_{1}^{\mu}$ and $s_{2}^{\mu}$ ). Additionally, they obey a partial parallel transport equation 32,

$$
P_{\nu}^{\mu} \frac{\mathrm{Ds}_{\mathrm{A}}^{\nu}}{\mathrm{d} \lambda}=0
$$

for a screen projection operator $P^{\mu \nu}=g^{\mu \nu}+U^{\mu} U^{\nu}-$ $d^{\mu} d^{\nu}$. Written in $3+1$ form in synchronous gauge, the evolution equations for the screen vectors become

$$
\begin{aligned}
\frac{\mathrm{d}}{\mathrm{dt}} s_{A}^{\mu}= & s_{A}^{j} V^{i}\left(\gamma_{j k} \frac{d}{d t} V^{k}-3 V^{k} K_{j k}+V_{l} V^{k} \Gamma_{k j}^{l}\right) \\
& -\Gamma_{k l}^{i} V^{k} s_{A}^{l}+\gamma^{i j} K_{j k} s_{A}^{k} .
\end{aligned}
$$

In practice, the term including the time derivative of $V^{k}$ is determined by the left-hand side of Eq. 14. The computed beam area should be invariant under rotations of the screen vectors in screen space, though not invariant under time-dependent rotations. Therefore, a good test for code validation is to vary the initial screen vectors and ensure there is no effect on the optical scalars.

In practice, we substitute the definition of $\theta$ in terms of $\ell$, and also define a variable analogous to the root solid angle subtended by the beam according to an observer, $\varphi=\mathrm{d} \ell / \mathrm{d} \lambda$ in Eq. 21 (at an observer, $\mathrm{d} \ell_{o b s} / \mathrm{d} \lambda=\sqrt{\Omega_{o b s}}$, where $\Omega_{o b s}$ is the solid angle subtended by the beam). We write the real and complex pieces of $\sigma$ separately, and also write the equations in terms of coordinate time $t$. In order to further simplify the equations of motion for $\sigma$, we define $\bar{\sigma}=\ell^{2} \sigma$.

Significantly, the evolution of the screen vectors is not only useful for tracking the area of a beam; the polarization vectors of photons obey an almost identical set of equations. Thus the equations we present here may also provide a new technique for studying gravitational effects on polarization. The major difference between evolution is only that normalization of screen vectors is enforced throughout their evolution.

\section{B. CALCULATION OF THE WEYL SCALAR IN SYNCHRONOUS GAUGE}

The dominant computational expense in integrating these equations through an arbitrary spacetime will clearly come from computing Weyl tensor components. In our code we employ synchronous gauge, where $\alpha=1$ and $\beta^{i}=0$. There are in principle only ten independent components of the Weyl tensor that need to be calculated; however in this work we will not take full advantage of this, thus we compute 21 components of the Riemann tensor. It may be interesting to study how alternative formulations compare, but we do not pursue that idea in this work. In order to compute terms on the right-hand side of the evolution equations, we first write

$$
\begin{aligned}
\mathcal{W}= & -\frac{1}{2}\left(R_{\mu \nu \rho \sigma}-\left(g_{\mu[\rho} R_{\sigma] \nu}-g_{\nu[\rho} R_{\sigma] \mu}\right)\right. \\
& \left.+\frac{1}{3} R g_{\mu[\rho} g_{\sigma] \nu}\right)\left(s_{1}^{\mu}-i s_{2}^{\mu}\right) p^{\nu} p^{\rho}\left(s_{1}^{\sigma}-i s_{2}^{\sigma}\right) \\
= & -\frac{1}{2} R_{\mu \nu \rho \sigma}\left(s_{1}^{\mu}-i s_{2}^{\mu}\right) p^{\nu} p^{\rho}\left(s_{1}^{\sigma}-i s_{2}^{\sigma}\right)
\end{aligned}
$$

as all contractions with $g_{\mu \nu}$ cancel and the last line follows from $g_{\mu \nu}\left(s_{1}^{\mu}-i s_{2}^{\mu}\right)\left(s_{1}^{\nu}-i s_{2}^{\nu}\right)=1-1=0$.

In order to reduce this expression further, we will need to write the Riemann tensor in terms of $3+1$ variables,

$$
\begin{aligned}
{ }^{(4)} R_{i l m j}= & \frac{1}{2}\left(\gamma_{i j, l m}-\gamma_{j l, i m}-\gamma_{i m, l j}+\gamma_{m l, i j}\right) \\
& +\Gamma_{q i j} \Gamma_{l m}^{q}-\Gamma_{q i m} \Gamma_{j l}^{q}-K_{i m} K_{j l}+K_{i j} K_{m l} \\
{ }^{(4)} R_{i 0 m j}= & \partial_{j} K_{i m}-\partial_{m} K_{i j}+\left(\gamma_{q i, m}+\Gamma_{i m q}\right) K_{j}^{q} \\
& -\left(\gamma_{q i, j}+\Gamma_{i j q}\right) K_{m}^{q} \\
{ }^{(4)} R_{i 00 j}= & -{ }^{(3)} R_{i j}-K K_{i j}+8 \pi\left(S_{i j}-\frac{1}{2} \gamma_{i j}(S-\rho)\right)
\end{aligned}
$$

The term $\mathcal{W}$ can now be re-expressed so that a smaller number of terms need actually be computed. Naively, there are $4 \cdot 4 \cdot 4 \cdot 4=256$ terms in the original expression,

$$
R_{\alpha \mu \nu \beta} s_{\mathcal{A}}^{\alpha} k^{\mu} k^{\nu} s_{\mathcal{B}}^{\beta}
$$


for $\mathcal{A}, \mathcal{B} \in\{1,2\}$. To reduce the number of terms, writing the index combination $\alpha, \mu \equiv A$ as a single index running over all combinations of $\alpha$ and $\mu$, and denoting $s_{\mathcal{A}}^{\alpha} k^{\mu} \equiv$ $\varsigma_{\mathcal{A}}^{A}$ leads to some simplification:

$$
\begin{aligned}
\mathcal{W} & \supset-\frac{1}{2} R_{\alpha \mu \nu \beta} s_{\mathcal{A}}^{\alpha} k^{\mu} k^{\nu} s_{\mathcal{B}}^{\beta} \\
& \equiv \frac{1}{2} R_{A B} \varsigma_{\mathcal{A}}^{A} \varsigma_{\mathcal{B}}^{B} \\
& \equiv W_{\mathcal{A} \mathcal{B}}^{\Sigma} .
\end{aligned}
$$

Because the indices $\alpha$ and $\mu$ are antisymmetric under interchange, the indices $A$ and $B$ need not run over all values, but only

$$
A, B \in\{01,02,03,10,12,13,20,21,23,30,31,32\} \text {. }
$$

The antisymmetry further allows terms to be combined. Denoting the canonical ordering of elements $A_{c} \in\{01,02,03,12,13,23\}$ and reversed $A_{r} \in$ $\{10,20,30,21,31,32\}$, so $A \in A_{c} \cup A_{r}$,

$$
\begin{aligned}
R_{A B} \varsigma_{\mathcal{A}}^{A} \varsigma_{\mathcal{B}}^{B} & =R_{A_{c} B} \varsigma_{\mathcal{B}}^{B}\left(\varsigma_{\mathcal{A}}^{A_{c}}-\varsigma_{\mathcal{A}}^{A_{r}}\right) \\
& \equiv R_{A_{c} B} \varsigma_{\mathcal{B}}^{B}\left(2 \varsigma_{\mathcal{A}}^{\left[A_{c}\right]}\right)
\end{aligned}
$$

and similarly for $B$, so

$$
R_{A B} \varsigma_{\mathcal{A}}^{A} \varsigma_{\mathcal{B}}^{B}=4 R_{A_{c} B_{c}} \varsigma_{\mathcal{A}}^{\left[A_{c}\right]} \varsigma_{\mathcal{B}}^{\left[B_{c}\right]}
$$

Because $A_{c}$ runs over 6 terms, and because $R_{A B}$ is symmetric, only 21 total components of $R_{A B}$ need to be calculated in order to evaluate the sum, along with the 6 components in each of $\varsigma_{\mathcal{A}}^{\left[A_{c}\right]}$ and $\varsigma_{\mathcal{B}}^{\left[B_{c}\right]}$. The six $\varsigma_{\mathcal{A}}^{\left[A_{c}\right]}$ are

$$
\begin{aligned}
\varsigma_{\mathcal{A}}^{[01]}=-\frac{1}{2} s_{\mathcal{A}}^{1} p^{0} & \varsigma_{\mathcal{A}}^{[12]}=\frac{1}{2}\left(s_{\mathcal{A}}^{1} p^{2}-s_{\mathcal{A}}^{2} p^{1}\right) \\
\varsigma_{\mathcal{A}}^{[02]}=-\frac{1}{2} s_{\mathcal{A}}^{2} p^{0} & \varsigma_{\mathcal{A}}^{[13]}=\frac{1}{2}\left(s_{\mathcal{A}}^{1} p^{3}-s_{\mathcal{A}}^{3} p^{1}\right) \\
\varsigma_{\mathcal{A}}^{[03]}=-\frac{1}{2} s_{\mathcal{A}}^{3} p^{0} & \varsigma_{\mathcal{A}}^{[23]}=\frac{1}{2}\left(s_{\mathcal{A}}^{2} p^{3}-s_{\mathcal{A}}^{3} p^{2}\right)
\end{aligned}
$$

and similarly for $\varsigma_{\mathcal{B}}^{\left[B_{c}\right]}$. The vectors $s^{\mu}$ can be chosen freely, so long as only the beam area and opening angle are of interest, and they are chosen to be orthogonal to $p^{\mu}$ and the observer's line of sight, $V^{i}$. The sum should then contain the $6 \cdot 6=36$ terms,

$$
\frac{1}{2} \mathcal{W}_{\mathcal{A} \mathcal{B}}^{\Sigma}=\sum_{A_{c}, B_{c} \in\{01,02,03,12,13,23\}} R_{A_{c} B_{c} \varsigma_{\mathcal{A}}^{\left[A_{c}\right]} \varsigma_{\mathcal{B}}^{\left[B_{c}\right]}}
$$

which contains the 21 unique components of the Riemann tensor that can be evaluated using Eq.??. The source terms for the optical scalar equations are then written as

$$
\begin{aligned}
& \Re[\mathcal{W}]=\frac{1}{2}\left(\mathcal{W}_{11}^{\Sigma}-\mathcal{W}_{22}^{\Sigma}\right) \\
& \Im[\mathcal{W}]=-\mathcal{W}_{12}^{\Sigma} .
\end{aligned}
$$

